\title{
ESPECIES SILVESTRES
}

\section{Estudio sistémico de la anatomía e histología del chigüiro (Hydrochaeris hydrochaeris)}

Corredor M. José Ricardo ${ }^{1}$ MVZ MSc, Sejin Carlos P. ${ }^{2}$ MVZ, Esp, y González G. Mario ${ }^{3}$ MV

${ }^{1}$ Docente asistente área fisiología, Escuela de Ciencias Animales Unillanos, 2Docente asociado área anatomía, Escuela de Ciencias Animales Unillanos,

${ }^{3}$ Docente asociado área histología, Escuela de Ciencias Animales Unillanos jcorredormatus@gmail.com

\section{RESUMEN}

Introducción. El chigüiro (Hydrochaeris hydrochaeris), es una de las especies silvestres con mayor potencialidad para explotación técnica a nivel de zoocriaderos. En la Orinoquia colombiana, no existen publicaciones que plasmen descripciones detalladas y que estén adecuadamente documentadas y en forma sistémica de la totalidad de la anatomía de esta especie. Objetivo. Realizar un estudio sistémico detallado de la anatomía e histología del Hydrochaeeris hydrochaeris y plasmar la información en un documento descriptivo tipo atlas, para ponerlo a disposición de la comunidad científica. Metodología. El estudio se llevó a cabo en los Laboratorios de Anatomía e histología de la Escuela de Ciencias Animales de Unillanos. Se utilizaron diez ejemplares entre hembras y machos., los cuales se sacrificaron siguiendo el protocolo utilizado en el Laboratorio. Cada dos ejemplares se trataron con protocolos de conservación de cadáveres, elaborados a base de formol, fenol, etanol y glicerina, a diferentes concentraciones. La aplicación del protocolo se realizó con una bomba de inyección de fluidos. El volumen aplicado correspondió al $10 \%$ del peso de cada animal. Con cada sacrificio de ejemplar se tomaron muestras de tejidos para el estudio histológico, conservadas en formalina al $10 \%$, de acuerdo con los protocolos del laboratorio de histología, donde fueron procesadas. La disección se inició a los diez días, siguiendo la siguiente secuencia: Piel, músculos, vasos, nervios, etc., de cabeza, cuello, tórax, miembros torácicos, cavidad abdominal, pélvica, perineo y miembros 
pelvianos. Las disecciones y placas histológicas se documentaron con fotografías y diagramas explicativos, así como con la descripción de cada uno de ellos. Resultados. Se presentan por capítulos así: Generalidades, planos y regiones corporales, sistema óseo, Artrología y Sindesmologia, sistema muscular, Sistema Nervioso, sistema Cardiovascular; esplacnología, que incluye Sistema Respiratorio y Sistema Digestivo, Sistema Urogenital. Se describen detalladamente cada estructura ósea del esqueleto axil y el apendicular. En miología se detalla cada grupo muscular por regiones, dando mayor énfasis a miembros torácicos y pelvianos. Se describen e identifican los nervios emergentes del plexo braquial y lumbosacro y nervios más importantes. En cardiovascular se presentan los principales vasos arteriales y venosos y una descripción detallada del corazón. El sistema respiratorio muestra características importantes de la especie. El sistema digestivo, resalta la formula dentaria y la dimensión del ciego. El sistema urogenital destaca el poro urogenital o rectogenital, donde se ubica el ano, el pene y la vagina. De cada órgano se hicieron descripciones histológicas, que en general coinciden con la arquitectura histológica de otros mamíferos, incluyendo domésticos. Conclusiones.Se presenta por primera vez en la región de la Orinoquia y el país, un documento que contiene el conocimiento completo de la anatomía e histología de esta especie, en forma de atlas descriptivo, para consulta y estudio de los interesados.

Palabras clave: Artrología, Especie silvestre, esplacnología, macro-anatomía, osteología 


\section{Estudio histopatológico retrospectivo de los casos procesados de fauna silvestre en la Universidad de los Llanos en el período 2004-2011 (I período)}

González Rojas Y. M., Ochoa Amaya J. E. y González Paya G. Grupo de Investigaciones en Patología de Animales Domésticos y Silvestres GRIPADS, Facultad de Ciencias Agropecuarias y Recursos Naturales, Universidad de los Llanos, Kilómetro 12 Vía Puerto López, Villavicencio, Meta-Colombia.

\section{gussgp@hotmail.com}

\section{RESUMEN}

Introducción. La Orinoquia Colombiana, por su complejidad orográfica, heterogeneidad de relieve y variedad de climas y microclimas, es una de las regiones con mayor riqueza biológica y, por lo tanto, una de las más biodiversas del país. El estudio de las enfermedades que afectan a los animales silvestres es abordado por trabajos histopatológicos retrospectivos, permitiendo una aproximación a la realidad sobre las enfermedades que afectan a estas especies. Objetivo. Conocer y describir las patologías que afectan a especies de fauna silvestre, de acuerdo con los archivos del Laboratorio de Histopatología de la Escuela de Ciencias Animales de la Universidad de los Llanos, durante el periodo comprendido entre 2004 y 2011 . Metodología. Este trabajo se llevó a cabo en la sede Barcelona de la Universidad de los Llanos. Se realizó en el Laboratorio de Histopatología de la Escuela de Ciencias Animales, Programa Medicina Veterinaria y Zootecnia, Facultad de Ciencias Agropecuarias y Recursos Naturales. Un análisis histopatológico de secciones de tejido de los casos archivados de la casuística de animales silvestres fue hecho. Inicialmente se realizó un estudio descriptivo, con diagnóstico histopatológico, teniendo en cuenta el diagnóstico preliminar de necropsia, la especie animal, la localización o tejido(s) implicado(s), su etiología y la frecuencia de presentación de la patología. Los datos fueron analizados con estadística descriptiva, para establecer las patologías más frecuentes en cada especie, su etiología y el sistema orgánico más afectado. Resultados. La mayor frecuencia de presentación se registró en los mamíferos 37 casos equivalentes al $89 \%$ de la casuística, seguida por los reptiles con 4 casos $(9 \%)$ y de las aves con 1 caso (2\%). La mayor presentación de enfermedades 
fueron las de tipo infeccioso con 26 casos (62\%), las no infecciosas con 11 casos (26\%) y las neoplasias con 5 casos (12\%). las enfermedades infecciosas fueron clasificadas por sistemas y el sistema mayormente afectado fue el digestivo con 23 casos (53\%), seguido por el respiratorio con 6 casos (14\%), el reproductor con 4 casos $(9 \%)$, el cardiovascular con 3 casos $(7 \%)$, nervioso con 3 casos $(7 \%)$, urinario con 2 casos $(5 \%)$ y por último piel y tegumentos con 2 casos $(5 \%)$. Entre las patologías no infecciosas, el mayor número de casos correspondió a las enfermedades de origen tóxico con 4 casos (37\%), seguidas de las de tipo metabólico con 3 casos (27\%), las inflamatorias con 2 casos (18\%), las de origen traumático con 1 caso (9\%) y por último las neurogénicas con un caso (9\%). Para la clasificación de las neoplasias, en las de origen epitelial fue 1 caso (20\%) diagnosticado, en las de origen mesenquimal 4 casos (80\%), para un total de 5 casos. Conclusiones. Se concluyó que las especies silvestres más afectadas son mamíferos, las patologías de mayor presentación afectaron el sistema digestivo, seguido del sistema respiratorio .Se halló que el $62 \%$ de los casos correspondió a patologías de tipo infeccioso, el $27 \%$ de tipo no infeccioso y el $11 \%$ de tipo neoplásico y de estas, el $80 \%$ fueron de tipo mesenquimal y el $20 \%$ de tipo epitelial.

Palabras clave: histopatología fauna silvestre 


\title{
Características hematológicas de la lapa (Cuniculus paca) en el departamento del Meta
}

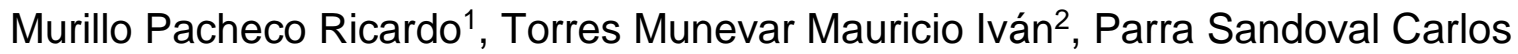 \\ Alberto $^{2}$ y José Fernández Manrique ${ }^{3}$ \\ ${ }^{1}$ Corporación Llanera de ornitología y de la naturaleza KOTSALA, \\ ${ }^{2}$ Corporación para el desarrollo sostenible del área de manejo especial la \\ Macarena-CORMACARENA, \\ ${ }^{3}$ Grupo Medicina Tropical, Programa MVZ, Universidad de los Llanos. \\ rimupa@yahoo.com
}

\section{RESUMEN}

Objetivo. Determinar las características hematológicas de la lapa Cuniculus paca, en el departamento del Meta, especie que presenta una carne de alto valor comercial, siendo una de las especies de mayor importancia alimentaria y económica en la región neo tropical. Para la determinación de las características hematológicas en $C$. paca, se utilizaron un total de 113 animales de ambos sexos, sin la presencia de signos clínicos. Antes de la toma de sangre, a cada ejemplar se le realizó una restricción química bajo la combinación de $50 \mathrm{~mL}$ de Zoletil®, 25 $\mathrm{mg}$ de Tiletamina y $25 \mathrm{mg}$ de Zolacepán en dosis de sedación de $2.5 \mathrm{mg} / \mathrm{Kg}$ y para anestesia en dosis de $5 \mathrm{mg} / \mathrm{Kg}$. La venipunción se efectuó mediante tubos vacutainer debidamente rotulados e identificados acoplados a agujas hipodérmicas calibre 21, recogiendo en un tubo $\sin$ anticoagulante $6 \mathrm{~mL}$ de sangre. Los parámetros evaluados fueron: hematocrito con un promedio de $41.18 \pm 7.83 \%$, hemoglobina de $23.18 \mathrm{~g} / \mathrm{dL}$, el VCM fue de $86.14 \mathrm{fL}$ y HCM de $29.85 \mathrm{pg}$, plaquetas o trombocitos, con $277.6 \pm 109.2 \mathrm{xmm}^{3}$, los linfocitos con un valor de $58.25 \pm 22.7 \%$, los monocitos con un promedio de $1.5 \pm 0.57 \%$. Un $7,07 \%$ presentó ligera policromatofilia. Estos valores comparados con otras especies de roedores presentan una relativa diferencia, confiriendo una repuesta diferente a la manipulación y al cautiverio de C. paca.

Palabras clave: Cuniculus paca, hematología, lapa, roedor. 